\title{
The neuroprotection of electro-acupuncture via the PGC-1a/TFAM pathway in transient focal cerebral ischemia rats
}

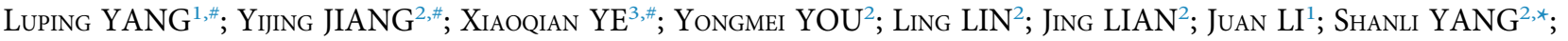 \\ XIEHUA XUE ${ }^{2, *}$ \\ ${ }^{1}$ School of Health Preservation and Rehabilitation, Chengdu University of Traditional Chinese Medicine, Chengdu, 610072, China \\ 2 Rehabilitation Hospital Affiliated to Fujian University of Traditional Chinese Medicine, Fuzhou, 350003, China \\ ${ }^{3}$ School of Acupuncture, Fujian University of Traditional Chinese Medicine, Fuzhou, 350003, China
}

Key words: Transient focal cerebral ischemia, Electro-acupuncture, PGC-1a/TFAM signaling pathway, ATP release

\begin{abstract}
ATP depletion is one of the pathological bases in cerebral ischemia. Electro-acupuncture (EA) is widely used in clinical practice for ischemia. However, the mechanism of EA remains unclear. The purpose of this study was to investigate whether EA could activate the AMPK/PGC-1 1//TFAM signaling pathway and, consequently, increase the preservation of ATP in rats with ischemia. In this study, 48 rats were randomly divided into four groups as a sham-operation control group (sham group), a middle cerebral artery occlusion group (MCAO group), an EA group, and an EA group blocked by the AMPK inhibitor compound C (EA + CC group) $(\mathrm{N}=12$ /group). The rats of the EA group and EA + CC group received the EA treatment for 7 days. The rats that belonged in the two remaining groups were only grasped in the same condition. Then, their brain tissues were collected for further detection. When compared with other groups, EA significantly reduced neurological deficits score and increased motor function. The cerebral infarction volume was significantly reduced in the EA group according to TTC staining. With western blot, we found that EA improved the ratio of p-AMPKa/AMPKa $(P<0.05)$, however, there is no difference between the MCAO group and sham group $(P>$ 0.05). In addition, EA also increased the expression of PGC-1 $\alpha$ and TFAM (all $P<0.05$ ). By Elisa, we observed that EA increased the preservation of ATP $(P<0.05)$ and mitochondrial respiratory enzymes, including Complex I $(P<0.05)$, Complex IV $(P<0.05)$, but not Complex III $(P>0.05)$. In summary, we conclude that EA may protect against ischemic damage in MCAO rats, improve the preservation of ATP and mitochondrial respiratory enzymes. This effect may be positively regulated by the activation of the PGC-1 1 /TFAM signaling pathway.
\end{abstract}

\section{Introduction}

Stroke is currently the second cause of adult death worldwide (Naghavi et al., 2017) and the leading cause of disability (Johnson et al., 2019). Ischemic stroke, which accounts for 85\% of all strokes (Gu et al., 2014), often leads to infarctions in the cortex, consequently causes neurological deficits and motor dysfunction. Acupuncture is a classical method in traditional Chinese medicine (TCM) and is widely applied in clinical practice for stroke patients. With that being said, Electro-acupuncture (EA) is a unique technique, featured as an electrical stimulation that is added to traditional acupuncture (Wu et al., 2017). Furthermore, clinical studies

*Address correspondence to: Shanli Yang, yangshanli@aliyun.com; Xiehua Xue, xuexiehua@aliyun.com

${ }^{\#}$ These authors contributed equally to this work

Received: 14 November 2020; Accepted: 22 March 2021 have reported that EA treatment is an effective and safe therapy for patients with stroke (Witt et al., 2009; Yu et al., 2019). However, the molecular mechanism of EA for stroke is still unclear.

As we have known, the brain is a high energy-consuming organ that greatly depends on adenosine triphosphate (ATP), which is responsible for supplying energy to maintain brain function. Once ATP depletion occurs, cell damage in the brain can lead to cell death (Fagundes et al., 2018; Giannone et al., 2012). Hence, it can consequently lead to both neurological deficit and motor dysfunction. ATP synthesis is correlated with mitochondrial respiratory chain (MRC), which is composed of $\mathrm{NADH}$ dehydrogenase (Complex I), succinate dehydrogenase (Complex II), cytochrome c reductase (Complex III), and cytochrome c oxidase (Complex IV) (Brown et al., 2010; Sousa et al., 2018). A previous study (Olivia et al., 2003) has found that the inhibition of mitochondrial respiratory chain Complexes I-III 
(MRCC I-III) resulted in a decrease of ATP in ischemia. In addition, other studies (Jornayvaz and Shulman, 2010; Scarpulla, 2008) have demonstrated that several protein factors were involved in the mitochondrial respiratory functions, such as peroxisome proliferator-activated receptor $\gamma$ coactivator $1 \alpha$ (PGC-1 $\alpha)$ and mitochondrial transcription factor A (TFAM). PGC-1 $\alpha$ is considered the initiating factor of mitochondrial respiratory functions (Puigserver and Spiegelman, 2003; Valero, 2014). It could upregulate the TFAM so as to maintain mitochondrial respiratory functions by promoting ATP production. The PGC-1 $1 /$ TFAM pathway is suggested to be a critical molecular mechanism of mitochondrial respiratory functions. Some studies proved that AMP-activated protein kinase (AMPK) tended to regulate the PGC-1 $\alpha /$ TFAM pathway. For example, Li et al. (2016) reported that the repression of the AMPK/PGC-1a signaling pathway was involved in the pathological process of ischemia. Some researchers (Dai et al., 2017; Zhong et al., 2008) have discovered that EA could increase the $\mathrm{Na}^{+}-\mathrm{K}^{+}$-ATPase activity and adenosine levels in cerebral ischemia rats. Thus, current evidence indicates that EA treatment has the potential to improve ATP release after stroke.

As mentioned above, we proposed that EA treatment may increase ATP release through the activation of the AMPK/PGC-1 $\alpha /$ TFAM signaling pathway in rats with ischemic stroke. Ultimately, it may improve both neurological deficit and motor dysfunction. To verify the hypothesis and to investigate the mechanism of EA treatment, we performed this experiment using an animal model of occlusion of the middle cerebral artery (MCAO). Besides, we observed the effect of EA on neurological deficit score, behavioral assessment, infarct volume, ATP and MRC level, and protein expression of AMPK, p-AMPK, PGC- $1 \alpha$, and TFAM.

\section{Materials and Methods}

Reagents and chemicals

Stainless acupuncture needles were prepared by Hua-Tuo Medical Appliance Factory, Suzhou, China. Electroacupuncture apparatus were purchased from Nanjing, China. The suture used in the middle cerebral artery occlusion/reperfusion (MCAO/R) was purchased from Guangzhou Duling Company, China. The primary reagents and chemicals were listed in
Tab. 1. The primary and secondary antibodies used in this study were listed in Tab. 2.

\section{Experimental animals and groups}

48 male Sprague-Dawley rats [6-8 weeks, 230-250 g, certificate No. SXCK (Zhe) 2019-002] were housed in standard cages under controlled temperature $\left(24 \pm 2^{\circ} \mathrm{C}\right)$ and a $12 \mathrm{~h} \mathrm{light/dark}$ cycle (light on 09:00-21:00). Food and water were free to obtain. Based on the random number tables, all the rats were divided into four groups $(\mathrm{N}=12$ /group): Sham-operated control group (sham group), MCAO group, MCAO + EA (EA group), $\mathrm{MCAO}+\mathrm{EA}+$ compound $\mathrm{C}$ group (EA + CC group). All experiments were conducted in accordance with the guidelines of the Institutional Animal Care and Use Committee of Fujian University of Traditional Chinese Medicine.

\section{Preparation of compound $C$}

Compound C (AMPK inhibitor; $10 \mathrm{mg} / \mathrm{kg}, 0.4 \mathrm{~mL} / 200 \mathrm{~g}$ ) was dissolved into $10 \%$ DMSO and divided into aliquots which were stored at $-20^{\circ} \mathrm{C}$ until use. Aliquots of compound $\mathrm{C}$ were administered intraperitoneally to rats in the EA + CC group at the onset of ischemia to inhibit AMPK activation (Yang et al., 2016).

\section{Surgery procedure}

Briefly, rats were anesthetized by $10 \%$ chloral hydrate (250 $\mathrm{mg} / \mathrm{kg}$ ). After making a ventral cervical midline incision, a nylon suture (diameter: $0.26 \mathrm{~mm}$ ) was gently introduced from the left common carotid artery (CCA) into the internal carotid artery (ICA), consequently blocking the origin of the middle cerebral artery. The incision was stitched timely. After $1.5 \mathrm{~h}$, the suture was withdrawn to achieve reperfusion. Then, all rats were placed in an environment at room temperature, and oral food intake was allowed. The CCA, ICA, and external carotid artery (ECA) of shamoperated rats were peeled off, but the bloodstream was not blocked. During the surgery, we used a body temperature keeping pad to maintain the body temperature of rats at $37^{\circ} \mathrm{C}$.

\section{Experimental treatment}

Rats of the EA group and EA + CC group received EA treatment at $48 \mathrm{~h}$ after reperfusion. The Stainless

TABLE 1

The primary reagents and chemicals

\begin{tabular}{lll}
\hline No. & Reagents and chemicals & Company \\
\hline 1 & $10 \%$ chloral hydrate & Shanghai Zhanyun Chemical Co., Ltd., Shanghai, China \\
2 & nylon suture & Guangzhou Duling Co., Ltd., Shanghai, China \\
3 & Stainless acupuncture needles & Suzhou Hua Tuo Medical Appliance Factory, Suzhou, China \\
4 & Electroacupuncture apparatus & HANS-200, Nanjing, China \\
5 & MRCC I Elisa Kit & Enzyme Immunoassay Industry of Jiangsu Co., Ltd., China. \\
6 & MRCC III Elisa Kit & Enzyme Immunoassay Industry of Jiangsu Co., Ltd., China. \\
7 & MRCC IV Elisa Kit & Enzyme Immunoassay Industry of Jiangsu Co., Ltd., China. \\
8 & ATP Elisa Kit & Enzyme Immunoassay Industry of Jiangsu Co., Ltd., China. \\
\hline
\end{tabular}

Note: MRRC: mitochondrial respiratory chain complex. 
TABLE 2

The primary and secondary antibodies

\begin{tabular}{llll}
\hline No. & Primary & Company & Dilution \\
\hline 1 & Phosphor-AMPKa (Thr172) Rabbit mAb & Cell Signaling Technology & $1: 1000$ \\
2 & AMPK alpha Mouse Monoclonal Antibody (66536-1-Ig) & Proteintech & $1: 1000$ \\
3 & PGC1a Mouse Monoclonal antibody (66369-1-Ig) & Proteintech & $1: 1000$ \\
4 & TFAM Rabbit Polyclonal antibody (22586-1-AP) & Proteintech & $1: 3000$ \\
5 & Beta Actin Mouse Monoclonal antibody(66009-1-Ig) & Proteintech & $1: 5000$ \\
6 & HRP-conjugated Affinipure Goat Anti-Mouse IgG(H+L) (SA00001-1) & Proteintech & $1: 10000$ \\
7 & HRP-conjugated Affinipure Goat Anti-Rabbit IgG(H+L) (SA00001-1) & Proteintech & $1: 10000$ \\
\hline
\end{tabular}

acupuncture needles ( $0.3 \mathrm{~mm}$ in diameter) were inserted into Qu chi (LI11), Shou san li (LI10), Zu san li (ST36), and Feng long (ST40) on the right limbs at 2-3 mm depth. EA apparatus was connected after acupuncture. Besides, EA treatment was given once a day for continuous 7 days, 30 min per time. EA stimulation using an interrupted wave and low frequency $(1-20 \mathrm{~Hz})$ was given to rats to induce the slight tremor of their limbs. Rats of the sham group and MCAO group were only grasped in the same condition. Rats were fixed using cloths during acupuncture.

\section{Evaluation of the neurological deficit scores}

Neurological evaluation was performed by the same researcher at $48 \mathrm{~h}$ after reperfusion and the $7^{\text {th }}$ day after treatment, who was blinded to the group assignment. In this experiment, we adopted the Longa test and modified neurological severity scoring (mNSS) to exam the neurological deficit. The Longa test is a five-point scale (Jiang et al., 2016): Grade 0, symptoms without neurological impairment (normal); Grade 1, inextensibility of its right forepaw when lifting the rats' tail(mild); Grade 2, circling to the right side while walking (moderate); Grade 3, walking hard and leaning to the right (severe); and Grade 4, cannot walk spontaneously (very severe). In addition, we also employed mNSS to evaluate the neurological deficit scores. The mNSS scale (Bieber et al., 2019; Sha et al., 2019) ranges from 0 (normal) to 18 (maximal deficit). The details of the mNSS test were shown in Appendix 1.

\section{Evaluation of the motor function \\ Slope test}

We used the slope test to assess the coordination ability and rapid-reaction capability. The rats were placed upside down on a $45^{\circ}$ inclined plane, and the time of turning their heads upward $>135^{\circ}$ was recorded. The results were calculated in seconds. The longer time indicated poorer reaction ability and coordination ability (Fig. $1\left(\mathrm{~A}^{1}-\mathrm{A}^{3}\right)$ ).

\section{Suspension test}

We used the suspension test to assess the muscle strength of the upper limbs in rats. The longer duration time indicated the stronger grasping ability of the upper limbs. Briefly, the rats were allowed to grasp a horizontal cylindrical tool $(0.5$ $\mathrm{cm}$ in diameter) with their front legs, and the height leaving from the ground was $4.5 \mathrm{~cm}$. The time when the rats fell from the tool was recorded. A 5-point scale was adopted in the test as follows: $0,<10 \mathrm{~s} ; 2,10-30 \mathrm{~s} ; 3,30 \mathrm{~s}-2 \mathrm{~min} ; 4$, 2-5 $\min ; 5$, $>5 \min$ (Fig. $1\left(\mathrm{~B}^{1}-\mathrm{B}^{3}\right)$ ).

\section{Balance beam test}

We used an elevated beam (1.5 m length, $25 \mathrm{~mm}$ width, and 15 $\mathrm{cm}$ height) to assess the gross motor function and balance ability of rats. Before the first test, rats received pre-trained to be familiar with the test. A 5-point scale was adopted in the test as follows (Liang et al., 2018): 0, the rat is able to keep balance and walk on the beam using its forelimbs symmetrically; 1 , the rat is able to keep balance and walk on the beam using its unaffected limb preferentially; 2, the rat is able to keep balance and walk on the beam mostly relying on the unaffected limb; 3 , the rat is not able to keep balance on the beam once moved; 4 , the rat fall off the beam immediately (Fig. $1\left(C^{1}-C^{3}\right)$ ).

\section{Measurement of cerebral infarct area}

Three rats from each group were sacrificed at $48 \mathrm{~h}$ after reperfusion and 7 days after EA treatment, respectively. All brains were taken quickly and frozen in a refrigerator at $-20^{\circ} \mathrm{C}$ for $20 \mathrm{~min}$. Then, the brain was cut into 5 slices (2 $\mathrm{mm}$ thickness) from the frontal extreme to the pillow pole with a sharp blade. In photophobic condition, the brain specimens were stained with $2 \%$ 2,3,5-triphenyl tetrazolium chloride (TTC) at $37^{\circ} \mathrm{C}$ for $20 \mathrm{~min}$ and fixed with $4 \%$ paraformaldehyde. The normal brain tissue stained red, while infarcted tissue unstained (white). The infarct volume was calculated with Swanson's method (Liu et al., 2009; Swanson et al., 1990). The total volumes of both contralateral and ipsilateral cortex were measured, and the infarct percentage was calculated as $\%$ contralateral structure.

\section{Enzyme-linked immunosorbent assay (ELISA) for} mitochondrial respiratory enzymes and ATP

Peri-infarct cortical tissue samples were resected from the MCAO group, EA group, and EA + CC group, using the method detailed by a previous study (Mätlik et al., 2014). The same position was obtained from the sham group. Brain tissue was obtained from the cortex of the rats prior to centrifugation at $1,000 \times g$ for $10 \mathrm{~min}$ at $4^{\circ} \mathrm{C}$. Ischemic brain tissue was cut into $30 \mathrm{mg}$ and collected in $270 \mu \mathrm{L}$ of phosphate-buffered saline (PBS). After homogenization by ultrasonic cell crusher (30 s, on ice), the tissue was centrifuged $\left(14,000 \times g, 4^{\circ} \mathrm{C}, 10 \mathrm{~min}\right)$ to collect the 

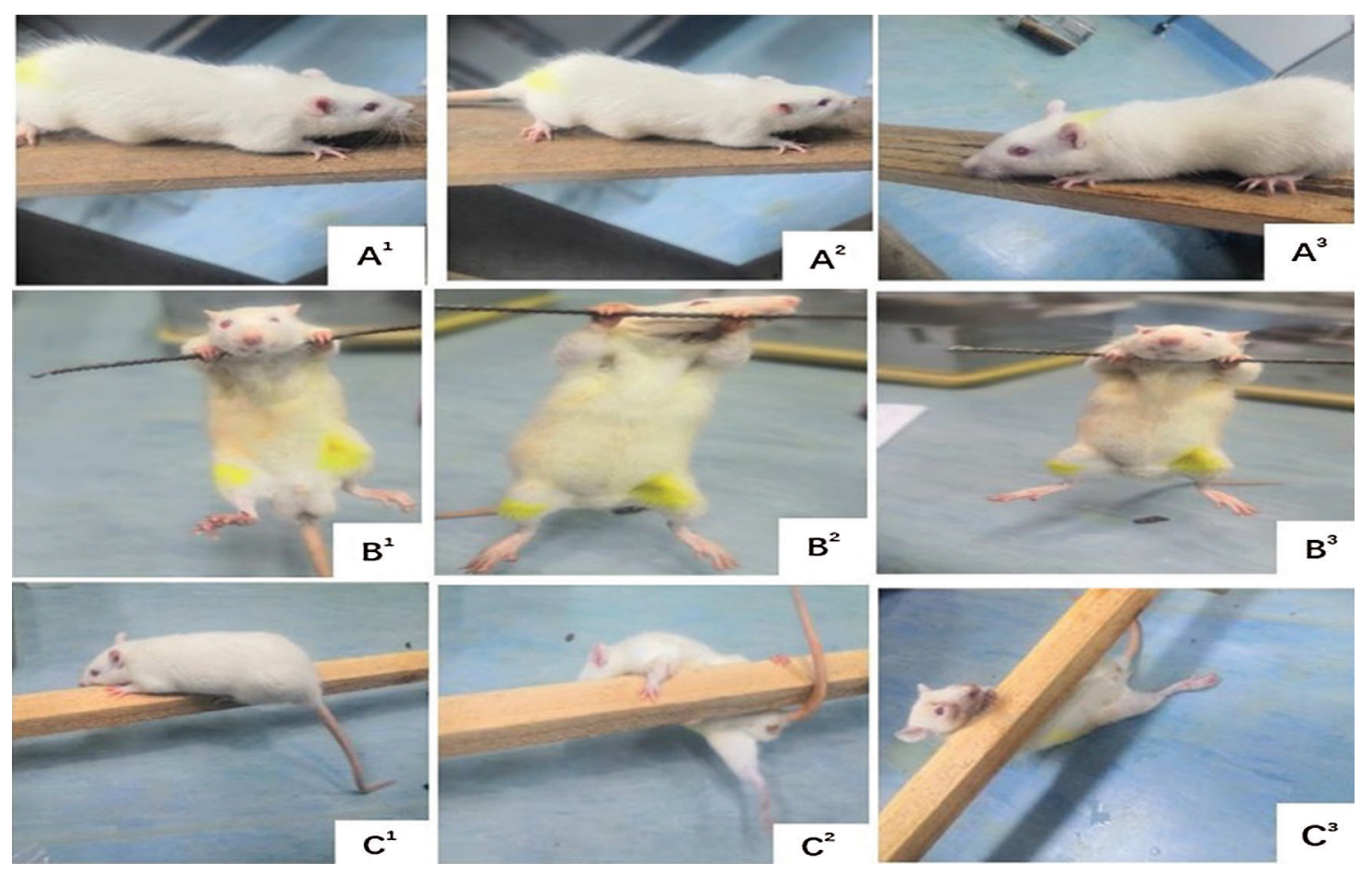

FIGURE

1.

Evaluation of the motor function in rats of each group. $\left(\mathrm{A}^{1}-\mathrm{A}^{3}\right)$ Slope test. $\left(B^{1}-B^{3}\right)$ Suspension test. $\left(\mathrm{C}^{1}-\mathrm{C}^{3}\right)$ Balance beam test

supernatant. The titers of AC, cAMP, and PKA were measured using Elisa, and the results were expressed as optical density (OD) units, according to previously published methods (35). The OD of each well was immediately determined using a microplate reader (Bio-Rad) set at $450 \mathrm{~nm}$.

\section{Western blot analysis}

We collected brain tissues in the peri-infarct cortical region from the MCAO group, EA group, and EA + CC group, and the same position from the sham group for western blot analysis. The brain tissue was homogenized by an ultrasonic cell crusher (30 s, on ice) and centrifuged $\left(14,000 \times g, 4^{\circ} \mathrm{C}\right.$, $10 \mathrm{~min}$ ) to collect the supernatant. BCA protein assay kit was used to assess the protein content. $50 \mu \mathrm{g}$ proteins extracted from each sample were separated by SDS-polyacrylamide gels electrophoresis (SDS-PAGE) and transferred to PVDF membranes. After blockage with 5\% nonfat milk for $2 \mathrm{~h}$ at room temperature, the membranes were incubated overnight at $4^{\circ} \mathrm{C}$ with a primary detection antibody. The dilution ratio of the primary detection antibody was listed in Tab. 2. Then, HRP-conjugated secondary antibody was added to the blots and incubated for $1 \mathrm{~h}$ at room temperature; protein bands were examined with Image Lab image acquisition and analysis software (Bio-Rad, Hercules, CA, USA). Gray values of proteins from each sample were shown as the densitometric ratio of proteins to $\beta$-actin.

\section{Statistical analysis}

SPSS statistical package (Version 20.0) was utilized to perform the statistical analysis and GraphPad Prism 8.0 for figures. All data were presented as the mean \pm standard deviation. Comparisons among multiple groups were analyzed with one-way ANOVA, followed by LSD (equal variances assumed) or Dunnett's T3 (equal variances not assumed) test for post hoc analysis. A $P$-value of less than 0.05 was considered statistically different. The flow chart of this experiment was shown in Fig. 2.

\section{Results}

\section{EA treatment reduced neurological deficits}

Before treatment, we observed that compared with the sham group, the neurological deficits score increased in the MCAO group, EA group, and EA + CC group (Longa test, all $P<0.05$; mNSS test, all $P<0.05$ ) after $48 \mathrm{~h}$ of focal cerebral ischemia, and no difference among the three groups (all $p>0.05$ ). The results indicated that the model of focal cerebral ischemia was successfully established. After treatment, EA significantly reduced neurological deficit scores (Longa test, $P<0.05$; mNSS test, $P<0.05$ ). There was no difference between the EA group and $\mathrm{EA}+\mathrm{CC}$ group $(P>0.05)$ in the Longa test, while a statistical difference was observed in the mNSS test $(P<0.05)$. Details are shown in Fig. 3. These results demonstrated that EA was able to improve the neurological deficits in transient focal cerebral ischemic rats.

\section{EA treatment increased motor function}

According to the results of the balance beam test, after $48 \mathrm{~h}$ focal cerebral ischemia, rats in the MCAO group, EA group, and EA + CC group showed poorer balance ability than sham group (all $P<0.05$ ). After treatment, compared with the MCAO group, EA treatment significantly improved the motor function of MCAO rats $(P<0.05)$ (Fig. 4A). We got the same results from the Slope test (Fig. 4B). However, there was no significant difference between groups based on the results of the suspension test $(P>0.05)$ (Fig. 4C). These results indicated that rats with focal cerebral ischemia exhibited motor disorder, and EA had the potential advantage to improve the coordination ability, rapidreaction capability, and balance ability in MCAO rats. 

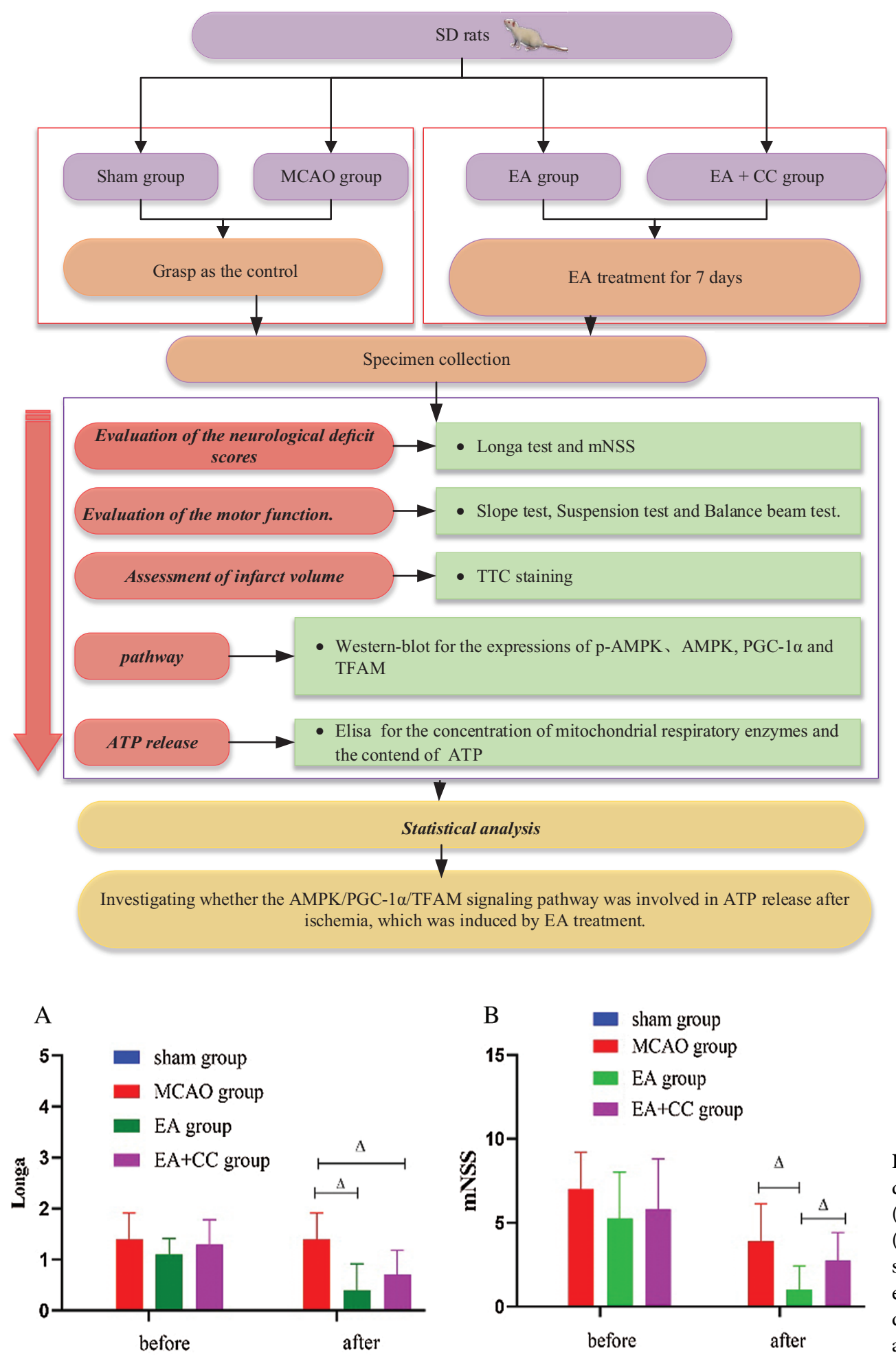

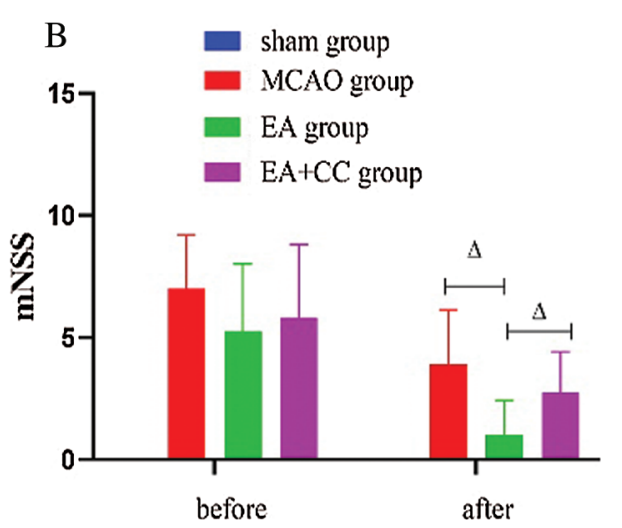

FIGURE 2. Flow chart showing the scheme of the experiment
EA treatment attenuated the infarct volumes of the rats' brains As shown in Fig. 5, at $48 \mathrm{~h}$ after reperfusion, there was no sign of cerebral injury in the sham group, while obvious cerebral infarction with white staining was obtained in all rats of the MCAO group, EA group, and EA + CC group (all $P<0.05$ ). No difference was existed among the MCAO group, EA group, and EA + CC group (all $P>0.05$ ). These results indicated that the MCAO model was successfully established. At 7 days after intervention, infarct volumes in the MCAO group were $47.93 \pm 6.92 \%$. In contrast, treating with EA significantly reduced infarct volume to $5.67 \pm 3.62 \%$
$(P<0.05)$. The infarct volume in EA + CC group decreased to $16.19 \pm 5.26 \%(P<0.05)$. However, we also noted that there is a marked decrease in cerebral infarct volumes in the MCAO group (from $77.8 \pm 11.33$ to $47.93 \pm 6.92$ ). The phenomenon may be associated with the tendency of selfhealing with the prolongation of ischemia time.

EA treatment increased the preservation of ATP and mitochondrial respiratory enzymes

As shown in Fig. 6, the concentration of mitochondrial respiratory enzymes, including Complex I, Complex III, 
A

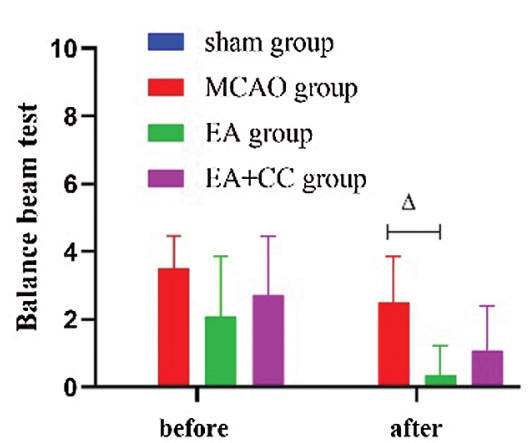

B

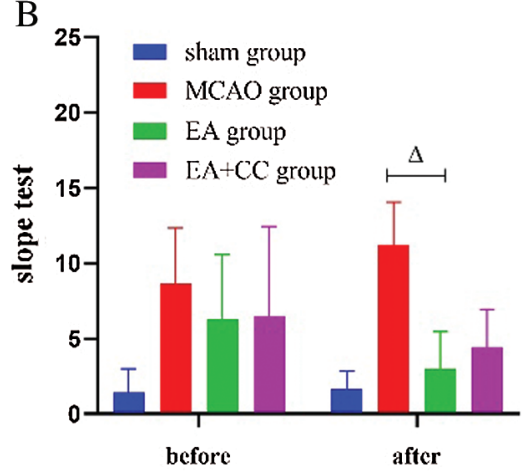

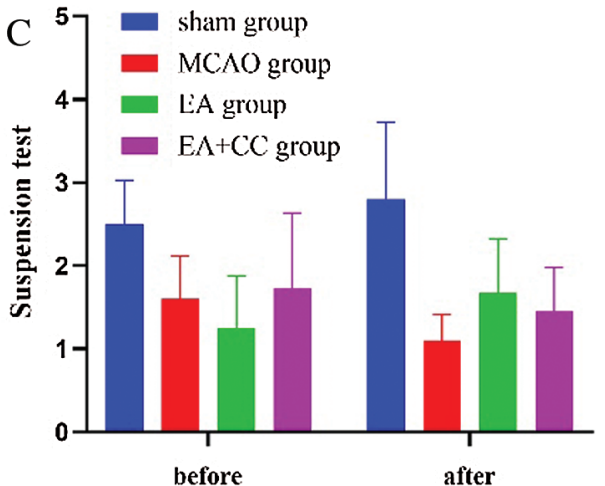

FIGURE 4. The results of motor function. (A) The results of the balance beam test; the score of the balance beam test in the sham group was 0 $(\mathrm{N}=9)$. (B) The results of the slope test $(\mathrm{N}=9)$. (C) The results of the suspension test $(\mathrm{N}=9)$. Error bars represented mean $\pm \mathrm{SD}$. ${ }^{\Delta} P<0.05$. EA: electro-acupuncture; CC: compound C.

A

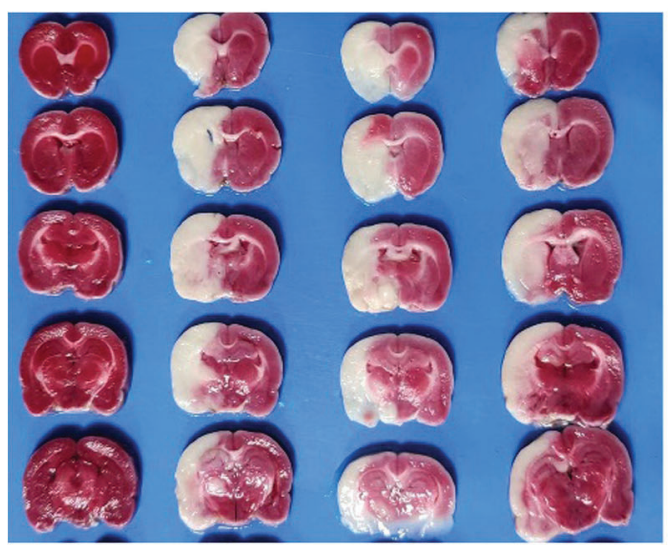

C

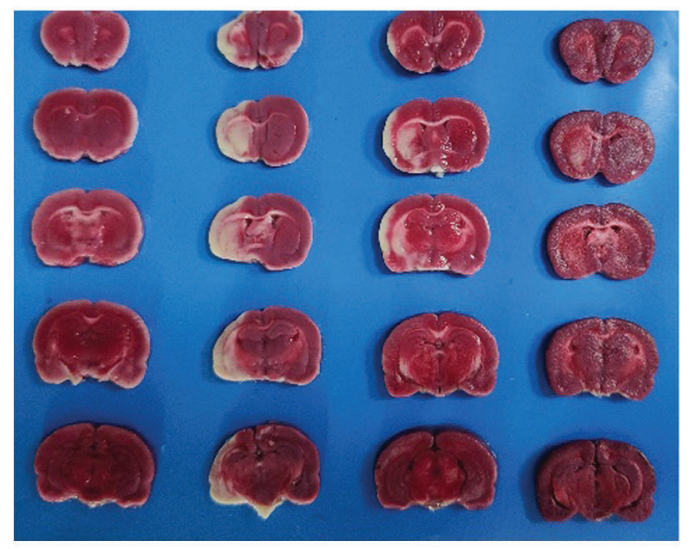

B

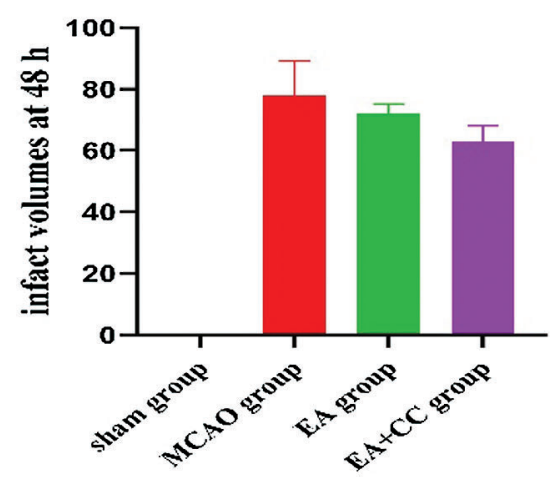

D

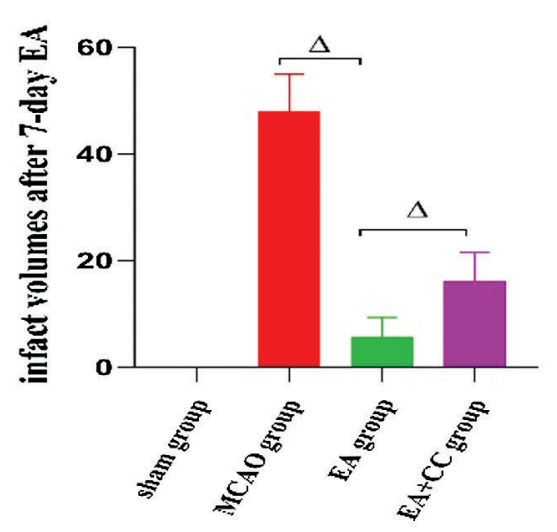

FIGURE 5. Effect of EA treatment on infarct volumes. The groups in A and $\mathrm{C}$ images, from left to right, are sham group, MCAO group, $\mathrm{EA}+\mathrm{CC}$ group, and EA group. (A) Representative graphs of TTCstained brain sections at $48 \mathrm{~h}$ after reperfusion $(\mathrm{N}=3)$. (B) Infarct volume quantitation of image $\mathrm{A}$ $(\mathrm{N}=3)$. (C) Representative graphs of TTC-stained brain sections after 7-day intervention $(\mathrm{N}=3)$ (D) Infarct volume quantitation of image $\mathrm{C}(\mathrm{N}=3)$. Error bars represented mean \pm SD. ${ }^{\Delta} P<0.05$. The infarct volume of the sham group was 0 . and Complex V, and the content of ATP were downregulated in MCAO rats (all $P<0.05$ ). Compared to the MCAO group, the EA group had a significant increase of the Complex I, Complex V, and ATP levels (all $P<$ 0.05), but not Complex III $(P>0.05)$. The concentration of these enzymes and the ATP content decreased in the $\mathrm{EA}+\mathrm{CC}$ group, and there was a significant difference between the EA group and EA + CC group $(P<0.05)$. All these results showed that ATP fall did exist in MCAO rats, and EA treatment was able to improve the preservation of ATP and mitochondrial respiratory enzymes.
EA treatment promoted ATP release through PGC-1 $\alpha / T F A M$ pathway in focal cerebral ischemia

As shown in Fig. 7, compared with the sham group, the ratio of $\mathrm{p}$-AMPKa/AMPKa, the expression level of PGC-1a and TFAM was downregulated in the MCAO group (p-AMPKa/AMPKa, $P>0.05$; PGC-1a, $P<0.05$; TFAM, $P<0.05)$. EA has a significant advantage to increase these indicators $(P<0.05)$, while compound $\mathrm{C}$ had an opposite influence on the ratio of p-AMPKa/AMPKa, the expression level of PGC-1a and TFAM $(P<0.05)$. These results demonstrated that EA treatment could regulate the PGC-1 $\alpha /$ TFAM pathway in focal cerebral ischemia. 
A

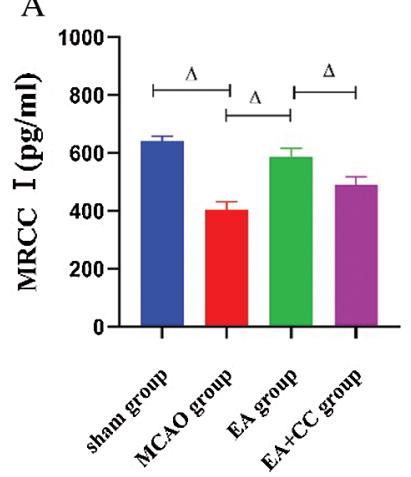

C

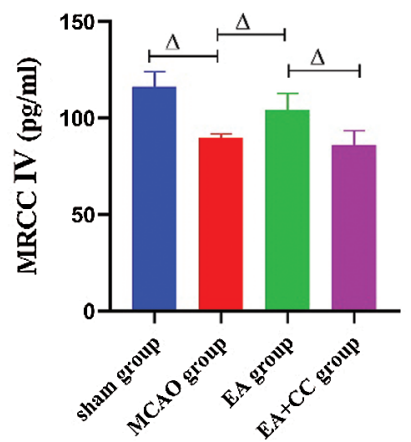

B

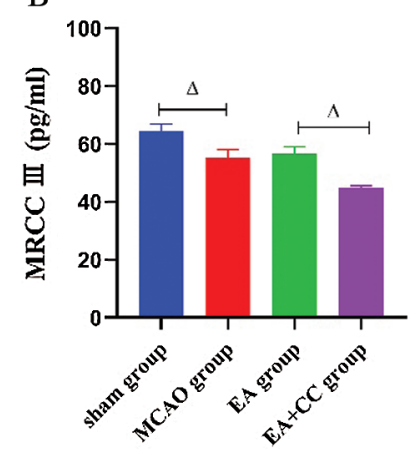

$\mathrm{D}$

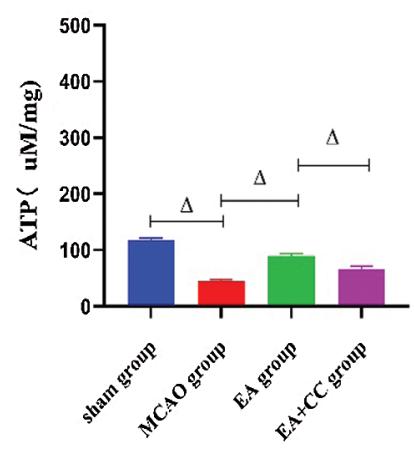

FIGURE 6. Effect of EA treatment on the concentration of mitochondrial respiratory enzymes and ATP release. The level of Complex I, Complex III, Complex IV, and ATP in brain tissue were measured using the ELISA test after the 7-day intervention (A-D). Data were expressed as mean $\pm \mathrm{SD}(\mathrm{N}=3) .{ }^{\Delta} P<0.05$. MRCC: mitochondrial respiratory chain complex.

\section{Discussion}

EA is popularly used in the treatment of cerebral ischemia. In clinical practice, EA is commonly performed with the acupoints of Quchi (LI11) and Zusanli (ST36) in patients with stroke (Chen et al., 2020; Cho et al., 2013). Several experimental studies have shown that the application of EA on Quchi (LI11) and Zusanli (ST36) acupoints, acted as a neuroprotective role in cerebral ischemic rats (Tao et al., 2016; Wu et al., 2017). In addition, some data mining studies (Zhang et al., 2018) suggested that the most frequently used meridian is the Yangming meridian. Thus, in this study, we chose Quchi (LI11), Zusanli (ST36), Shousanli (LI10), and Fenglong (ST40) and evaluated the effects of EA treatment in MCAO rats.

Firstly, our study showed that EA treatment could decrease the infarct volume, reduce the neurological deficit, and improve motor function in MCAO rats. Our findings were consistent with several other studies. Sha et al. (2019) and Liu and Lai (2006) reported that EA was able to decrease the infarct volume in MCAO rats. The improvement of motor function induced by EA treatment has been confirmed by animal experiments (Xu et al., 2015), clinical study (Wang et al., 2020b), and meta-analysis (Cai et al., 2017). However, we noted that the infarct volume also decreased in the MCAO group; the reason may be related to the self-healing capability after reperfusion in MCAO rats. Li et al. (2021) observed that the infarct volume of the MCAO group decreased 14 days after reperfusion, and they

reported spontaneous recovery existed in the brain lesions of the MCAO rats. Liu and Lai (2006) also observed that infarct volumes of MCAO rats presented a natural reduction between 3 and 7 days after reperfusion.

Secondly, we found that the ATP level, the MRCC concentration, and protein expression of PGC-1 $\alpha$ and TFAM significantly decreased in MCAO rats. The results indicated that the ATP depletion caused by the inactivation of the PGC-1 $\alpha$ /TFAM pathway may be one of the vital factors for focal cerebral ischemia. When cerebral ischemia occurs, a series of complex pathological events called the ischemic cascade (Brouns and Deyn, 2009; Hossmann, 2006) such as energy failure, neurotoxicity, peri-infarct depolarization, and programmed cell death, may happen. During this process, the energy failure caused by ATP depletion was an initiator of brain damage and ultimately lead to cell death (Frenguelli, 2019). Both Olivia et al. (2003) and Zhao et al. (2013) reported that the occlusion of the middle cerebral artery caused a great reduction of the ATP levels in MCAO rats. Additionally, MRCC, which is comprised of five complexes, could transport electrons to form a proton motive force and further regulate the ATP synthesis (Nagao et al., 2020). A robust increase in the content of the de-active form of Complex I (Stepanova et al., 2019) and Complex IV was observed in ischemic rats (Racay et al., 2009). PGC-1a, as a vital role in the regulation of energy metabolism in tissues/organs (Finck and Kelly, 2006; Handschin and Spiegelman, 2006; St-Pierre et al., 2006), could directly/ indirectly upregulate the TFAM. The PGC-1a/TFAM is a major upstream signaling pathway of MRCC (Fu et al., 2016; Yang et al., 2018). Wang et al. (2020a) found that low expression of PGC-1 $\alpha$ and TFAM protein led to a reduction of ATP, Complex I and V in MCAO rats. Geng et al. (2017) reported that poor expression of PGC-1 $\alpha$ decreased the ATP content, and PGC-1a may be a therapeutic target for the treatment of cerebral ischemia/reperfusion injury.

Thirdly, we discovered that the over-expression of PGC-1a and TFAM induced by EA may improve the preservation of ATP, Complex I, and Complex IV. Some researchers have proposed that preventing ATP depletion may be a neuroprotective strategy in brain ischemia. Our study confirmed this proposition and was consistent with the results of several studies. Researchers reported that the improvement of ATP and MRCC protected neurons in ischemia rats (Dai et al., 2017; Galeffi et al., 2010; Olivia et al., 2003). In addition, our results elucidated that EA activated the PGC-1a/TFAM signaling pathway in ischemic rats. Numerous studies have demonstrated that PGC- $1 \alpha$ is able to protect against cerebral ischemia. Xu et al. (2018) reported that the activation of PGC$1 \alpha$ could reduce the cerebral infarction volume and improve the neurological deficit in MCAO rats. Wang et al. (2016) found that over-expression of PGC-1 $\alpha$ and TFAM induced by carbon monoxide (CO) could promote ATP release and increase brain mitochondrial biogenesis in global cerebral ischemia. Therefore, the activation of the PGC-1a/TFAM signaling pathway might be a potential therapeutic target for ischemia. Liu et al. (2019) reported that EA significantly upregulated the expression of PGC- $1 \alpha$ and TFAM in heart tissue of myocardial ischemia rats. Xie (2016) also observed that the high expression of PGC-1 $\alpha$ and TFAM protein could 
A

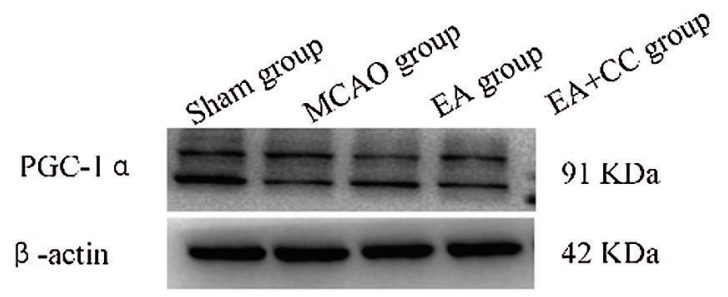

C

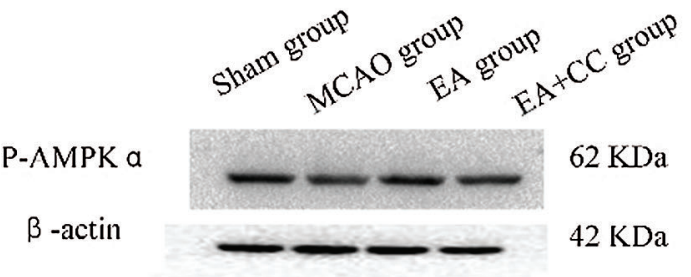

$\mathrm{E}$

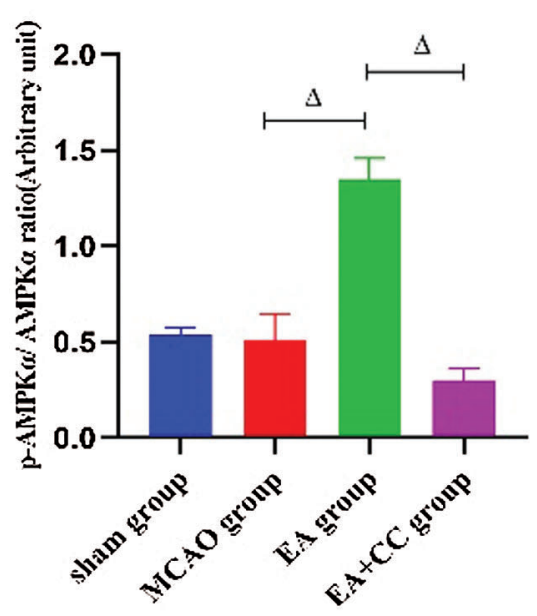

$\mathrm{F}$

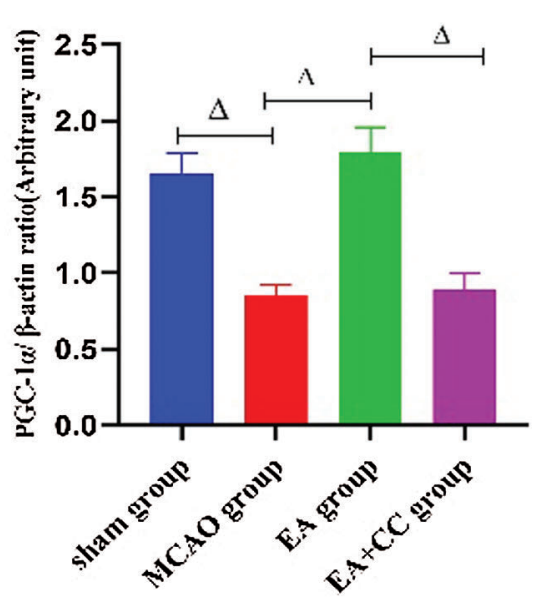

B

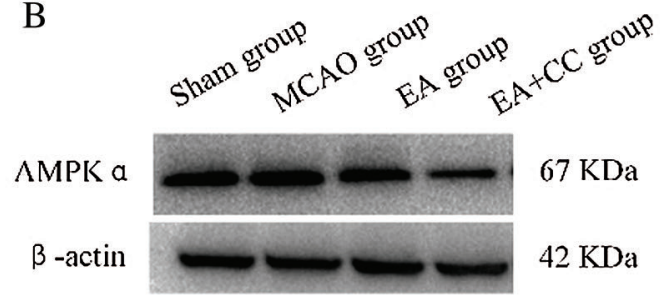

$\mathrm{D}$

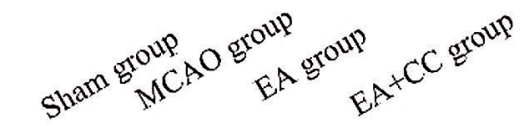

TFAM

$\beta$-actin

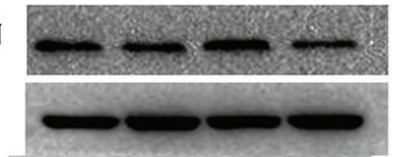

$27 \mathrm{KDa}$

$42 \mathrm{KDa}$
G

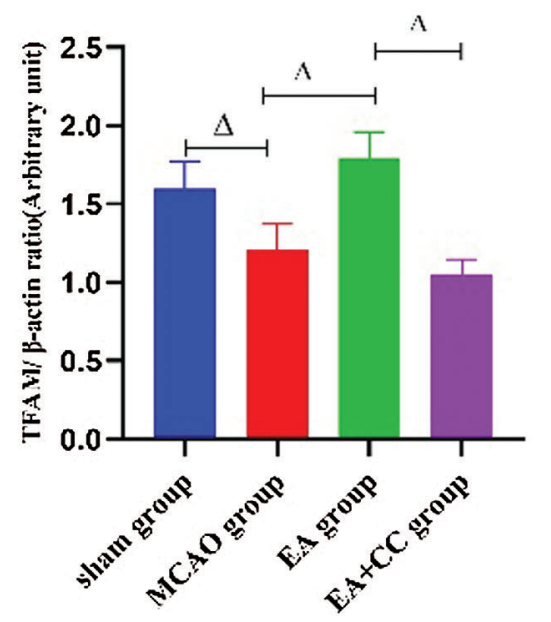

FIGURE 7. Effect of EA treatment on levels of marker proteins related to AMPK and PGC-1 $\alpha$ /TFAM pathway. (A-D) Representative images of PGC-1 $\alpha$, p-AMPKa, AMPKa and TFAM in the peri-infarct cortical region; (E-G) Western blot analysis of the expression of PGC-1 $\alpha$ and TFAM, and quantitative analysis of the ratio of p-AMPKa/AMPKa. $\beta$-actin as the internal control $(\mathrm{N}=3)$. Data were expressed as mean \pm SD. ${ }^{\Delta} P<0.05$.

be induced by EA in myocardial ischemia rats. Our study found EA upregulated the expression of PGC-1 $\alpha$ and TFAM in MCAO rats, indicated that EA may act as a neuroprotective role through activation of the PGC- $1 \alpha /$ TFAM signaling pathway.

However, we noted that no statistical difference between the MCAO group and sham group in the ratio of p-AMPK/AMPKa. While the behavioral results showed that the EA + CC group could alleviate neurological deficits and motor function. Probably, some other molecule regulates the PGC-1 $1 \alpha /$ TEAM pathway, and AMPK may be a concomitant consequence of EA in ischemia rather than a mechanistic cause.

Overall, our results revealed that EA could reduce infarct volume and neurological deficits, improve the motor function in focal cerebral ischemia rats via promoting the preservation of ATP and mitochondrial respiratory enzymes. This effect may be related to the activation of the PGC- $1 \alpha /$ TFAM pathway.

There were also some limitations in this study. Firstly, the sample size of the TTC staining, ELISA test, and Western blot was small. Secondly, the behavioral assessment of rats was limited by the subjective judgment of the researchers. More objective assessment tools are required to reduce the assessment bias, such as the catwalk system (Caballero-Garrido et al., 2017) or the homecage scan monitoring system (Salem et al., 2016). Thirdly, a more intuitive device could be utilized to estimate whether the model was established, such as laser-Doppler flowmetry (LDF) (Harada et al., 2005). Fourthly, intraventricular injection is optimum for further research study. Fifthly, EA's placebo effect should be taken into consideration, and sham acupuncture should be added as a comparison.

\section{Conclusion}

Our results suggested that EA could protect against ischemic damage in MCAO rats, improve the preservation of ATP and mitochondrial respiratory enzymes. Thus, this effect may be positively regulated by the activation of the PGC-1 $\alpha /$ TFAM signaling pathway.

Acknowledgement: We would like to appreciate Sashanna Ruth Kalynna Mcvean for English language copy editing our manuscript.

Availability of Data and Materials: The datasets generated during and/or analysed during the current study are available from the corresponding author on reasonable request. 
Authors' Contributions: The authors confirm contribution to the paper as follows: study conception and design: Luping Yang, Yijing Jiang; the experiment: Luping Yang, Xiaoqian Ye, Yongmei You, Ling Lin, Jing Lian, analysis and interpretation of results: Luping Yang, Juan Li; draft manuscript preparation: Luping Yang; manuscript revision: Shanli Yang, Xiehua Xue. All authors reviewed the results and approved the final version of the manuscript.

Ethics Approval: The study was approved by the Ethics Committee of Fujian University of Traditional Chinese Medicine on May 10, 2019. The paper involving rats, their care of the laboratory animals complied with Guidance Suggestions for the Care and Use of Laboratory Animals 2006 administered by Ministry of Science and Technology, China.

Funding Statement: The present study was supported by the National Natural Science Foundation of China (No. 81574048 and No. 81904268) and Key personnel training project of Health Care in middle-aged and young people in Fujian province (No. 2019-ZQN-81).

Conflicts of Interest: The authors declare that they have no competing interests.

\section{References}

Bieber M, Gronewold J, Scharf AC, Schuhmann MK, Langhauser F et al. (2019). Validity and reliability of neurological scores in mice exposed to middle cerebral artery occlusion. Stroke 50: $2875-2882$.

Brouns R, Deyn PPD (2009). The complexity of neurobiological processes in acute ischemic stroke. Clinical Neurology \& Neurosurgery 111: 483-495.

Brown GC, Murphy MP, Rich PR, Maréchal A (2010). The mitochondrial respiratory chain. Essays in Biochemistry 47: 1-23.

Caballero-Garrido E, Pena-Philippides JC, Galochkina Z, Erhardt E, Roitbak T (2017). Characterization of long-term gait deficits in mouse dMCAO, using the CatWalk system. Behavioural Brain Research 331: 282-296.

Cai Y, Zhang CS, Liu S, Wen Z, Zhang AL, Guo X, Lu C, Xue CC (2017). Electroacupuncture for poststroke spasticity: A systematic review and meta-analysis. Archives of Physical Medicine and Rehabilitation 98: 2578-2589.

Chen C, Yu Q, Xu K, Cai L, Felicia BM et al. (2020). Electroacupuncture pretreatment prevents ischemic stroke and inhibits Wnt signaling-mediated autophagy through the regulation of GSK-3 $\beta$ phosphorylation. Brain Research Bulletin 158: 90-98.

Cho SY, Kim M, Sun JJ, Jahng GH, Kim HJ, Park SU, Jung WS, Ko CN, Park JM (2013). A comparison of brain activity between healthy subjects and stroke patients on fMRI by acupuncture stimulation. Chinese Journal of Integrative Medicine 19: 269-276.

Dai QX, Geng WJ, Zhuang XX, Wang HF, Mo YC, Xin H, Chen JF, Wang JL (2017). Electroacupuncture-induced neuroprotection against focal cerebral ischemia in the rat is mediated by adenosine A1 receptors. Neural Regeneration Research 12: 228-234.

Fagundes DJ, Carrara FL, Teixeira WA, Simões RS, Taha MO (2018). The role of the exogenous supply of adenosine triphosphate in the expression of Bax and Bcl2L1 genes in intestinal ischemia and reperfusion in rats 1. Acta Cirugica Brasileira 33: 889-895.

Finck BN, Kelly DP (2006). PGC-1 coactivators: Inducible regulators of energy metabolism in health and disease. Journal of Clinical Investigation 116: 615-622.

Frenguelli BG (2019). The purine salvage pathway and the restoration of cerebral ATP: Implications for brain slice physiology and brain injury. Neurochemical Research 44: 661-675.

Fu XF, Yao K, Du X, Li Y, Yang XY, Yu M, Li MZ, Cui QH (2016). PGC-1 $\alpha$ regulates the cell cycle through ATP and ROS in $\mathrm{CH} 1$ cells. Journal of Zhejiang University-Science B 17: 136-146.

Galeffi F, Sinnar S, Schwartz-Bloom RD (2010). Diazepam promotes ATP recovery and prevents cytochrome $c$ release in hippocampal slices after in vitro ischemia. Journal of Neurochemistry 75: 1242-1249.

Geng H, Li Y, Shi Z, Li Y, Wang L (2017). Over-expression of PGC-1a reverses mitochondrial function reduction and apoptosis in OGD/R-induced neurons. Chinese Journal of Pathophsiology 33: 2078-2083.

Giannone FA, Treré D, Domenicali M, Grattagliano I, Baracca A et al. (2012). An innovative hyperbaric hypothermic machine perfusion protects the liver from experimental preservation injury. Scientific World Journal 2012: 573410.

Gu S, Sun Y, Han R, Wang L, Wang D, Wang J, Li X (2014). Association between genetic polymorphisms of cytochrome P450 2C19 and the risk of cerebral ischemic stroke in Chinese. BMC Medical Genetics 15: 83.

Handschin C, Spiegelman BM (2006). Peroxisome proliferatoractivated receptor gamma coactivator 1 coactivators, energy homeostasis, and metabolism. Endocrine Review 27: 728-735.

Harada H, Wang Y, Mishima Y, Uehara N, Makaya T, Kano T (2005). A novel method of detecting rCBF with laserDoppler flowmetry without cranial window through the skull for a MCAO rat model. Brain Research Brain Research Protocols 14: 165-170.

Hossmann KA (2006). Pathophysiology and therapy of experimental stroke. Cellular \& Molecular Neurobiology 26: 1055-1081.

Jiang Y, Yang S, Tao J, Lin Z, Ye X, You Y, Peng J, Hong Z, Chen L (2016). Opposing needling promotes behavior recovery and exerts neuroprotection via the cAMP/PKA/CREB signal transduction pathway in transient MCAO rats. Molecular Medicine Reports 13: 2060-2070.

Johnson CO, Nguyen M, Roth GA, Nichols E, Alam T et al. (2019). Global, regional, and national burden of stroke, 1990-2016: A systematic analysis for the Global Burden of Disease Study 2016. Lancet Neurology 18: 439-458.

Jornayvaz FR, Shulman GI (2010). Regulation of mitochondrial biogenesis. Essays in Biochemistry 47: 69-84.

Li L, Xiao L, Hou Y, He Q, Zhu J, Li Y, Wu J, Zhao J, Yu S, Zhao Y (2016). Sestrin2 silencing exacerbates cerebral ischemia/ reperfusion injury by decreasing mitochondrial biogenesis through the AMPK/PGC-1a pathway in rats. Scientific Reports 6: 30272.

Li Z, Yang M, Lin Y, Liang S, Liu W, Chen B, Huang S, Li J, Tao J, Chen L (2021). Electroacupuncture promotes motor function and functional connectivity in rats with ischemic stroke: An animal resting-state functional magnetic resonance imaging study. Acupuncture in Medicine 39: 146-155.

Liang D, He XB, Wang Z, Li C, Gao BY, Wu JF, Bai YL (2018). Remote limb ischemic postconditioning promotes motor 
function recovery in a rat model of ischemic stroke via the up-regulation of endogenous tissue kallikrein. CNS Neuroscience \& Therapeutics 24: 519-527.

Liu F, Schafer DP, Mccullough LD (2009). TTC, fluoro-Jade B and NeuN staining confirm evolving phases of infarction induced by middle cerebral artery occlusion. Journal of Neuroscience Methods 179: 1-8.

Liu L, Zhang D, Qu Y, Dong J, Wang J, Yong R, Xue Y, Deng T (2019). Effect of electroacupuncture at Zusanli (ST) on expression of PGC-1 alpha/NRFs/TFAM signaling pathway in myocardium of rats with spleen Qi deficiency. Chinese Archives of Traditional Chinese Medicine 36: 282.

Liu Z, Lai X (2006). Effect of electroacupuncture on neurological deficit and cerebral infarction volume in MCAO rats. Chinese Journal of Basic Medicine in Traditional Chinese Medicine 12: 456-456.

Mätlik K, Abo-Ramadan U, Harvey BK, Arumäe U, Airavaara M (2014). AAV-mediated targeting of gene expression to the peri-infarct region in rat cortical stroke model. Journal of Neuroscience Methods 236: 107-113.

Nagao T, Shintani Y, Hayashi T, Kioka H, Kato H et al. (2020). Higdla improves respiratory function in the models of mitochondrial disorder. FASEB Journal 34: 1859-1871.

Naghavi M, Abajobir AA, Abbafati C, Abbas KM, Abd-Allah F et al. (2017). Global, regional, and national age-sex specific mortality for 264 causes of death, 1980-2016: A systematic analysis for the Global Burden of Disease Study 2016. The Lancet 390: 1151-1210.

Olivia H, Javier DC, Verónica S, Ignacio L, Antonio C, Pereira MP, Isabel CM, Leza JC, Pedro L, María MA (2003). Inhibition of glutamate release by delaying ATP fall accounts for neuroprotective effects of antioxidants in experimental stroke. Faseb Journal Official Publication of the Federation of American Societies for Experimental Biology 17: 2082.

Puigserver P, Spiegelman BM (2003). Peroxisome proliferatoractivated receptor-gamma coactivator 1 alpha (PGC-1 alpha): Transcriptional coactivator and metabolic regulator. Endocrine Reviews 24: 78-90.

Racay P, Tatarkova Z, Chomova M, Hatok J, Kaplan P, Dobrota D (2009). Mitochondrial calcium transport and mitochondrial dysfunction after global brain ischemia in rat hippocampus. Neurochemical Research 34: 1469-1478.

Salem G, Krynitsky J, Kirkland B, Lin E, Chan A, Anfinrud S, Anderson S, Garmendia-Cedillos M, Belayachi R, AlonsoCruz J (2016). Scalable vision system for mouse homecage ethology. International Conference on Advanced Concepts for Intelligent Vision Systems.

Scarpulla RC (2008). Transcriptional paradigms in mammalian mitochondrial biogenesis and function. Physiological Reviews 88: 611-638.

Sha R, Zhang B, Han X, Peng J, Zheng C, Zhang F, Huang X (2019). Electroacupuncture alleviates ischemic brain injury by inhibiting the miR-223/NLRP3 pathway. Medical Science Monitor: International Medical Journal of Experimental and Clinical Research 25: 4723-4733.

Sousa JS, D’imprima E, Vonck J (2018). Mitochondrial respiratory chain complexes, In: Harris J, Boekema E (eds.), Membrane Protein Complexes: Structure and Function. Subcellular Biochemistry. Singapore: Springer.

St-Pierre J, Drori S, Uldry M, Silvaggi JM, Rhee J et al. (2006). Suppression of reactive oxygen species and neurodegeneration by the PGC-1 transcriptional coactivators. Cell 127: 397-408.
Stepanova A, Konrad C, Guerrero-Castillo S, Manfredi G, Vannucci S, Arnold S, Galkin A (2019). Deactivation of mitochondrial Complex I after hypoxia-ischemia in the immature brain. Journal of Cerebral Blood Flow and Metabolism: Official Journal of the International Society of Cerebral Blood Flow and Metabolism 39: 1790-1802.

Swanson RA, Morton MT, Tsao-Wu G, Savalos RA, Davidson C, Sharp FR (1990). A semiautomated method for measuring brain infarct volume. Journal of Cerebral Blood Flow and Metabolism: Official Journal of the International Society of Cerebral Blood Flow and Metabolism 10: 290-293.

Tao J, Zheng Y, Liu W, Yang S, Huang J, Xue X, Shang G, Wang X, Lin R, Chen L (2016). Electro-acupuncture at LI11 and ST36 acupoints exerts neuroprotective effects via reactive astrocyte proliferation after ischemia and reperfusion injury in rats. Brain Research Bulletin 120: 14-24.

Valero T (2014). Mitochondrial biogenesis: pharmacological approaches. Current Pharmaceutical Design 20: 5507-5509.

Wang J, Duan J, Huang S, Hu D, Chen H, Mu F (2020a). ElabelaAPLNR inhibits cerebral ischemia/reperfusion injury through AMPK/SIRT3/PGC-1 $\alpha$ and Akt/Nrf2 pathways. Research Square.

Wang P, Yao L, Zhou LL, Liu YS, Chen MD et al. (2016). Carbon monoxide improves neurologic outcomes by mitochondrial biogenesis after global cerebral ischemia induced by cardiac arrest in rats. International Journal of Biological Sciences 12: 1000-1009.

Wang Z, Lin Z, Zhang Y, Gao X, Robinson N (2020b). Motor entry point acupuncture for shoulder abduction dysfunction after stroke: A randomized controlled feasibility trial. European Journal of Integrative Medicine 35: 101073.

Witt CM, Pach D, Brinkhaus B, Wruck K, Tag B, Mank S, Willich SN (2009). Safety of acupuncture: Results of a prospective observational study with 229,230 patients and introduction of a medical information and consent form. Forschende Komplementarmedizin 16: 91-97.

Wu J, Lin B, Liu W, Huang J, Shang G, Lin Y, Wang L, Chen L, Tao J (2017). Roles of electro-acupuncture in glucose metabolism as assessed by 18F-FDG/PET imaging and AMPKa phosphorylation in rats with ischemic stroke. International Journal of Molecular Medicine 40: 875-882.

Xie J (2016). Effect of electroacupucture with "combined biao and ben acupoints" in myocardial ischemia and its signalling pathway in mitochondrail [Doctoral Dissertation]. Wuhan: Hubei University of Traditional Chinese Medicine.

Xu Q, Yang JW, Cao Y, Zhang LW, Zeng XH, Li F, Du SQ, Wang LP, Liu CZ (2015). Acupuncture improves locomotor function by enhancing GABA receptor expression in transient focal cerebral ischemia rats. Neuroscience Letters 588: 88-94.

Xu Y, Kabba JA, Ruan W, Wang Y, Zhao S, Song X, Zhang L, Li J, Pang T (2018). The PGC-1 $\alpha$ activator ZLN005 ameliorates ischemia-induced neuronal injury in vitro and in vivo. Cellular and Molecular Neurobiology 38: 929-939.

Yang JL, Mukda S, Chen SD (2018). Diverse roles of mitochondria in ischemic stroke. Redox Biology 16: 263-275.

Yang Y, Zhang XJ, Li LT, Cui HY, Zhang C et al. (2016). Apelin-13 protects against apoptosis by activating AMP-activated protein kinase pathway in ischemia stroke. Peptides 75: 96-100.

Yu CC, Ma CY, Wang H, Kong LH, Zhao Y, Shen F, Wu M (2019). Effects of acupuncture on Alzheimer's disease: Evidence from neuroimaging studies. Chinese Journal of Integrative Medicine 25: 631-640. 
Zhang Y, Zhang Z, Wang X (2018). Based on data mining technology, the acupuncture and moxibustion treatment of hemiplegia spasm state after stroke was studied. Inner Mongolia Traditional Chinese Medicine, 58-60.

Zhao Q, Wang S, Li Y, Wang P, Li S, Guo Y, Yao R (2013). The role of the mitochondrial calcium uniporter in cerebral ischemia/ reperfusion injury in rats involves regulation of mitochondrial energy metabolism. Molecular Medicine Reports 7: 1073-1080.

Zhong G, Li Z, Li W, Wang SE, Tang H, Deng G, Liu Y (2008). The influence of electro- acupuncture on contents of $\mathrm{Ca}^{2+}$ and $\mathrm{Na}^{+}-\mathrm{K}^{+}$-ATPase activity of mitochondria in rats with cerebral ischemia and reperfusion injury. Cuiding Journal of TCM 14: 12-14 [in Chinese].

Appendix 1

\section{The details of mNSS test}

\begin{tabular}{|c|c|c|c|}
\hline Context & & Details & Points \\
\hline \multirow[t]{4}{*}{ Motor tests } & \multirow[t]{3}{*}{ Raising rat by the tail } & Flexion of forelimb & 1 \\
\hline & & Flexion of hindlimb & 1 \\
\hline & & Head moved $>10^{\circ}$ to vertical axis within $30 \mathrm{~s}$ & 1 \\
\hline & \multicolumn{2}{|c|}{$\begin{array}{l}\text { Placing rat on the floor (normal }=0 \text {; maximum }=3 \text { ): } \\
0 \text { : Normal walk } \\
\text { 1: Inability to walk straight } \\
\text { 2: Circling toward the paretic side } \\
\text { 3: Fall down to the paretic side }\end{array}$} & $0-3$ \\
\hline Sensory tests & \multicolumn{2}{|c|}{$\begin{array}{l}\text { 1: Placing test (visual and tactile test). } \\
\text { 2: Proprioceptive test (deep sensation, pushing the paw against the table edge to } \\
\text { stimulate limb muscles). }\end{array}$} & $0-2$ \\
\hline Beam balance tests & $\begin{array}{l}\text { (normal }=0 \text {; maximun } \\
\text { 0: Balances with steady } \\
\text { 1: Grasps side of beam } \\
\text { 2: Hugs the beam and } \\
\text { 3: Hugs the beam and } \\
\text { 4: Attempts to balance } \\
\text { 5: Attempts to balance } \\
\text { 6: Falls off: No attemp }\end{array}$ & $\begin{array}{l}\text { b falls down from the beam } \\
\text { bs fall down from the beam, or spins on beam }(>60 \mathrm{~s}) \\
\text { beam but falls off }(>20 \mathrm{~s}) \\
\text { ance or hang on to the beam }(<20 \mathrm{~s})\end{array}$ & $0-6$ \\
\hline \multirow[t]{4}{*}{ Reflexes absent and abnormal movements } & \multicolumn{2}{|c|}{ Pinna reflex (head shake when touching the auditory meatus) } & 1 \\
\hline & \multicolumn{2}{|c|}{ Corneal reflex (eye blink when lightly touching the cornea with cotton) } & 1 \\
\hline & \multicolumn{2}{|c|}{ Startle reflex (motor response to a brief noise from snapping a clipboard paper } & 1 \\
\hline & \multicolumn{2}{|c|}{ Seizures, myoclonus, myodystonia } & 1 \\
\hline Maximum points & \multicolumn{2}{|l|}{$0-18$} & \\
\hline
\end{tabular}

